\section{Questionários indicam um baixo efeito global do nível de atividade física e do controle do estresse sobre o perfil lipídico de homens}

\author{
Questionnaires suggest a small overall effect of physical \\ activity level and stress control over men's lipid profile
}

\section{Maria Fátima Glaner ${ }^{1}$}

Resumo - Objetivou-se verificar se o perfil lipídico (PL) difere entre homens classificados em duas categorias de nível de atividade física (NAF) e de controle do estresse; e, analisar os resultados à luz das evidências que apontam relação inversa entre NAF, PL e controle de estresse (ctrl stress). A amostra foi de 109 sujeitos (idade $=33,7 \pm 8,0$ anos). Para o NAF utilizou-se o Questionário de Atividades Físicas Habituais, paro o ctrl stress o questionário do Perfil do Estilo de Vida Individual, sendo os sujeitos estratificados, respectivamente, em uma das categorias: "NAF ruim" ou "NAF bom", "ctrl stress sim" ou "ctrl stress não". O PL foi obtido por espectrofotometria enzimática. A análise de variância a dois fatores $(p<0,05)$ indicou que o PF não diferiu $(p>0,05)$ entre os homens com NAF bom e ruim, e que possuem, ou não, ctrl stress. A chance do NAF e do ctrl stress influenciarem o PL foi baixa ( $>00,05)$. Para o NAF, oscilou de 14,5\% (VLDL) a 23,7\% (LDL), para o ctrl stress de $5,1 \%(\mathrm{LDL})$ a $10,9 \%$ (VLDL). Ambos explicaram muito pouco ( $\mathrm{p}>0,05)$ a variação do $\mathrm{PF}$, sendo a mais alta de $1,7 \%$. O efeito global do NAF e do ctrl stress pode ser generalizado para todos os PL, quando usados os mesmos instrumentos do presente estudo. Estes achados têm implicações importantes quando o propósito for averiguar se os sujeitos alcançaram, ou não, o NAF bom. Hipotetiza-se que tais achados são devidos a possível falta de acuracidade na obtenção do NAF e do ctrl stress.

Palavras-chave: Atividade física; Atividade motora; Colesterol; Dislipidemia; Estresse da vida; Questionários.

Abstract - This study aimed to verify whether lipid profile (LP) differs between men classified into two categories of physical activity level (PAL) and stress control; and to analyze the results according to the evidence that suggests an inverse relationship among PAL, LP and stress control. The sample was composed of 109 subjects (age $=33.7 \pm 8.0$ years). To evaluate the PAL we used the "Habitual Physical Activity Questionnaire". To evaluate stress control, we used the "Profile of Individual Lifestyle" questionnaire, classifying subjects into one of the following categories: "bad PAL" or "good PAL", "yes stress control" or "no stress control". We obtained the LP using an enzymatic spectrophotometric method. Analysis of variance with two factors $(p<0.05)$ indicated that the LP did not differ $(p>0.05)$ between men in the "bad PAL" / "good PAL" groups and in the "yes stress control" / "no stress control" groups. There was a small chance $(p>0.05)$ for PAL and stress control to have an influence on $L P$, ranging from 14.5 (VLDL) to 23.7\% (LDL) for PAL and from 5.1 (LDL) to $10.9 \%$ $(V L D L)$ for stress control. These factors hardly explained $(p>0.05)$ the LP variation, which peaked at $1.7 \%$. When under the same circumstances of this study, the overall effect of PAL and stress control may apply to all LP. These findings have important implications for studies that aim to verify whether the subjects reached "good PAL". We suppose that these findings are due to a possible inaccuracy in the PAL and stress control assessment.

Key words: Cholesterol; Dyslipidemia; Life stress; Motor activity; Physical activity; Questionnaires.
1 Universidade Estadual de Londrina, Centro de Educação Física e Esportes, Departamento de Educação Física, Londrina. PR, Brasil.

Recebido em 24/09/12 Revisado em 11/01/13 Aprovado em 21/03/13 


\section{INTRODUÇÃO}

Como consequência da evolução brusca do desenvolvimento industrial e tecnológico, mazelas como o sedentarismo se instalaram e têm aumentado a cada década. Estudos têm apontado uma relação de causa e efeito entre uma série de fatores de risco e doenças crônicas não transmissíveis, principalmente as cardiovasculares, com o sedentarismo ou baixos níveis de atividade física ${ }^{1,2}$. Nesse contexto, tradicionalmente, a atividade física é operacionalizada como o gasto energético acima das condições de estado em repouso. Quanto maior for o gasto energético maior será a proteção contra determinados fatores de risco para doenças, principalmente, as crônicas não transmissíveis ${ }^{1}$.

Estudos clássicos, feitos em diferentes épocas, revelaram que sujeitos sedentários apresentam perfil lipídico inadequado, com altos valores de colesterol total, lipoproteínas de baixa densidade, glicemia e baixos valores de lipoproteínas de alta densidade ${ }^{3-5}$. Ainda, a atividade física praticada regularmente por toda a vida é fortemente associada com a redução de todas as causas de mortalidade em sujeitos ativos comparados aos sedentários ${ }^{1}$.

Em proporção muito menor, estudos indicam que a prática regular de atividades físicas, em níveis moderado ou intenso, também apresenta associação no controle do estresse, da ansiedade e dos demais sintomas depressivos, aumentando a transmissão sináptica das endorfinas ${ }^{6,7}$. Isto pode sugerir a influência positiva da atividade física regular na atenuação do desencadeamento do processo de estresse em adultos ${ }^{8}$. Nessa ótica, a atividade física praticada com regularidade é uma forma simples e eficaz para reduzir ou controlar o estresse.

Conforme já exposto, de um lado, há uma série de evidências sobre os benefícios da prática regular de atividade física moderada ou intensa, do outro, estudos apontam altas prevalências de sedentarismo em todas as regiões do Brasil ${ }^{9,10}$.

Ainda, tanto o nível de atividade física (NAF) quanto o controle do estresse são geralmente caracterizados via questionários. Evidências apontam a falta de acuracidade destes instrumentos ${ }^{11,12} \mathrm{e}$, além disso, o NAF é menos associado aos fatores de risco cardiovascular do que o consumo máximo de oxigênio ${ }^{13}$.

Por um lado, com frequência se tem observado estudos que analisaram a capacidade de determinadas variáveis, de fácil mensuração e baixo custo (ex. índice de massa corporal, perímetro do abdômen) ${ }^{14,15}$, em predizer fatores de risco cardiovascular. Por outro, há carência de estudos com o propósito de averiguar se o NAF e o controle de estresse, estimados por questionário, são capazes de diferenciar sujeitos quanto ao perfil lipídico, um dos principais fatores de risco cardiovascular.

Nesse contexto, este estudo tem como objetivo primário verificar se o perfil lipídico difere entre homens classificados em duas categorias de nível de atividade física (bom, ruim) e de controle do estresse (sim, não) e; como secundário, analisar os resultados obtidos à luz das evidências que apontam relação inversa entre o NAF, o perfil lipídico e o controle de estresse. 


\section{PROCEDIMENTOS METODOLÓGICOS}

\section{Amostra}

A amostra inicial foi composta por 200 funcionários de uma indústria metalúrgica do Distrito Federal. Os critérios de exclusão aplicados foram: não responder a todas as questões dos questionários ou haver alguma resposta inválida; não respeitar o jejum alimentar de 12h; usar repositores hormonais orais ou injetáveis, remédios antilipêmicos e para o controle do diabetes; fumar ou ingerir bebidas alcoólicas de forma regular (mais do que dois dias na semana); apresentar mal-estar agudo como vertigens ou febre, ou crônico, como esclerose das veias dos braços; triglicérides $\geq 400$ $\mathrm{mg} / \mathrm{dL}$; possuir algum tipo de metal no corpo (prótese, pino, marca-passo). Considerados esses critérios, a amostra final foi composta por 109 homens.

\section{Coleta de dados}

O estudo foi aprovado pelo Comitê de Ética da Universidade Católica de Brasília, parecer No. 04/2005. Previamente ao início da pesquisa, todos os voluntários foram esclarecidos sobre a proposta do estudo e assinaram um termo de consentimento livre e esclarecido.

Os dados foram coletados em duas etapas. Na primeira os voluntários responderam a um questionário de reconhecimento da amostra. Logo após, as suas variáveis antropométricas foram mensuradas. Em seguida foram submetidos ao exame da absortometria de raio X de dupla energia (AXDE). Na segunda etapa (entre dois a sete dias após a primeira), inicialmente, sob orientação, responderam aos questionários. Posteriormente foi realizada a coleta sanguínea na enfermaria da metalúrgica.

\section{Antropometria}

A massa corporal (MC) e a estatura (ES) foram mensuradas conforme a padronização descrita em Lohman et al. ${ }^{16}$, com fins de caracterizar a amostra e alimentar o software do exame de AXDE. A gordura corporal relativa (G\%) foi mensurada por meio da AXDE, modelo Lunar, DPX-IQ, software v. 4.7e. Para tanto, os voluntários retiraram os calçados e todos os objetos metálicos que estivessem portando. Em seguida, posicionavam-se em decúbito dorsal na área de leitura do aparelho, com os pés unidos e os braços estendidos ao longo do tronco. Assim permaneceram até o término do exame. A partir da análise de corpo inteiro foi obtida a G\%. Todas as medidas foram realizadas seguindo rigorosamente as instruções descritas no manual do referido equipamento. Todas as análises foram realizadas por um técnico experiente que apresenta coeficiente de variação $<1 \%$ para exames de corpo inteiro.

\section{Atividade física e controle do estresse}

O nível de atividade física (NAF) foi estimado por meio do Questionário de Atividades Físicas Habituais (QAFH), traduzido e adaptado por $\mathrm{Nahas}^{17}$. Este instrumento possibilita a classificação dos sujeitos em quatro 
categorias. Aqueles que foram classificados de sedentários a pouco ativos compuseram o grupo "NAF ruim", enquanto que os moderadamente ativos a muito ativos compuseram o grupo "NAF bom".

O controle do estresse (ctrl stress) foi identificado por meio do questionário do Perfil do Estilo de Vida Individual ${ }^{15}$. O grupo que controla seu estresse -"ctrl stress sim"- foi composto pelos sujeitos que apresentaram comportamento "quase sempre ou sempre verdadeiro no seu dia-a-dia", em atividades como: reservar ao menos $5 \mathrm{~min}$ por dia para relaxar; manter uma discussão sem se alterar, mesmo quando contrariado; equilíbrio entre o tempo dedicado ao trabalho e ao lazer. Aqueles que apresentaram comportamento oposto nestas atividades formaram o grupo "ctrl stress não".

\section{Perfil lipídico}

As variáveis do perfil lipídico foram quantificadas a partir de uma amostra $(3 \mathrm{~mL})$ de sangue venoso, a qual foi colhida por meio da punção de uma das artérias da fossa antecubital do braço. A coleta ocorreu no período da manhã, com os voluntários em jejum de $12 \mathrm{~h}$. A glicemia, o colesterol total (CT), as lipoproteínas de alta densidade (HDL - do inglês: high density lipoprotein), muito baixa densidade (VLDL - do inglês: very low-density lipoprotein) e os triglicérides foram quantificados em um espectrofotômetro semiautomático (Bioplus: BIO-2000) usando reagentes bioquímicos Doles . Foram seguidas as instruções do manual do equipamento e das bulas dos reagentes. As lipoproteínas de baixa densidade (LDL - do inglês: low density lipoprotein) foram calculadas pela equação de Friedewald et al. ${ }^{18}$. O índice aterogênico é o quociente do CT pelas HDL .

\section{Análises estatísticas}

A estatística descritiva foi utilizada para caracterizar a amostra nas variáveis mensuradas. Os resultados são apresentados em valores médios com os respectivos desvios padrões ( \pm ). A análise de variância a dois fatores foi usada para verificar se o perfil lipídico difere entre homens classificados em duas categorias de NAF (bom, ruim) e de ctrl stress (sim, não). As análises foram realizadas no programa Statistical Package for the Social Science (SPSS), versão 14.0, sendo considerada a chance de $5 \%(\mathrm{p}<0,05)$ para a rejeição da hipótese nula.

\section{RESULTADOS}

O grupo apresentou características heterogêneas quanto à idade, estatura, massa corporal e gordura corporal relativa (Tabela 1), indicando que os resultados podem ser generalizados para os sujeitos com ampla variabilidade nestas.

Os valores médios e os respectivos desvios padrões do perfil lipídico, dos homens classificados em duas categorias de nível de atividade física e de controle de estresse, estão apresentados na Tabela 2. A comparação via 
análise de variância a dois fatores resultou nos valores apresentados na Tabela 3. Os resultados indicam que não ocorreram diferenças ( $p>0,05)$, nas variáveis do perfil lipídico, entre homens com NAF bom e ruim e que possuem, ou não, controle do estresse. O poder do teste indicou uma probabilidade muito baixa das atividades para o controle do estresse $(5,1 \%$ a $8,6 \%)$ e do NAF $(14,5 \%$ a $23,2 \%)$ influenciar o perfil lipídico. Consequentemente, também não ocorreu interação $(\mathrm{p}>0,05)$ entre o NAF e o ctrl de stress ( $\mathrm{NAF}^{*}$ ctrl stress) para nenhuma das variáveis do perfil lipídico.

Tabela 1. Idade e características antropométricas da amostra $(n=109)$.

\begin{tabular}{lccc}
\hline Variáveis & $\bar{x}$ & Mínimo & Máximo \\
\hline Idade (anos) & $33,7 \pm 8,0$ & 20 & 51 \\
Estatura (cm) & $168,0 \pm 6,8$ & 155,5 & 189,5 \\
Massa corporal (kg) & $71,8 \pm 9,4$ & 55,1 & 95,0 \\
Gordura (\%) & $20,0 \pm 8,1$ & 6,0 & 37,1 \\
\hline
\end{tabular}

Tabela 2. Valores médios (mg/dL) do perfil lipídico em relação ao nível de atividade física (NAF) e controle do estresse (Ctrl stress).

\begin{tabular}{|c|c|c|c|c|c|}
\hline NAF & ctrl stress & $n$ & Colesterol total & HDL & LDL \\
\hline \multirow[t]{3}{*}{ Ruim } & Não & 17 & $160,3 \pm 33,3$ & $36,6 \pm 8,8$ & $98,1 \pm 36,6$ \\
\hline & Sim & 13 & $178,3 \pm 22,2$ & $39,6 \pm 6,5$ & $110,5 \pm 26,6$ \\
\hline & Total & 30 & $163,9 \pm 31,7$ & $37,2 \pm 8,3$ & $100,6 \pm 34,5$ \\
\hline \multirow[t]{3}{*}{ Bom } & Não & 57 & $159,3 \pm 35,4$ & $41,3 \pm 11,6$ & $96,8 \pm 30,3$ \\
\hline & Sim & 22 & $152,5 \pm 34,6$ & $42,1 \pm 14,4$ & $86,5 \pm 30,2$ \\
\hline & Total & 79 & $157,4 \pm 34,9$ & $41,6 \pm 12,3$ & $93,9 \pm 30,3$ \\
\hline \multirow[t]{4}{*}{ Total } & Não & 74 & $159,6 \pm 34,5$ & $39,9 \pm 11,0$ & $97,2 \pm 31,9$ \\
\hline & Sim & 35 & $158,0 \pm 33,6$ & $41,6 \pm 13,0$ & $91,6 \pm 30,5$ \\
\hline & Total & 109 & $159,1 \pm 34,0$ & $40,4 \pm 11,5$ & $95,7 \pm 31,4$ \\
\hline & & & VLDL & Triglicérides & IA \\
\hline \multirow[t]{3}{*}{ Ruim } & Não & 17 & $26,2 \pm 13,3$ & $131,1 \pm 66,6$ & $4,72 \pm 1,75$ \\
\hline & Sim & 13 & $28,4 \pm 3,9$ & $141,8 \pm 19,3$ & $4,59 \pm 0,94$ \\
\hline & Total & 30 & $26,6 \pm 12,0$ & $133,2 \pm 59,8$ & $4,69 \pm 1,60$ \\
\hline \multirow[t]{3}{*}{ Bom } & Não & 57 & $21,5 \pm 13,4$ & $109,7 \pm 69,4$ & $4,14 \pm 1,50$ \\
\hline & Sim & 22 & $25,4 \pm 13,9$ & $123,2 \pm 67,9$ & $3,97 \pm 1,43$ \\
\hline & Total & 79 & $22,6 \pm 13,51$ & $113,5 \pm 68,6$ & $4,09 \pm 1,47$ \\
\hline \multirow[t]{3}{*}{ Total } & Não & 74 & $22,9 \pm 13,4$ & $116,0 \pm 68,7$ & $4,31 \pm 1,58$ \\
\hline & Sim & 35 & $26,0 \pm 12,5$ & $127,1 \pm 60,9$ & $4,10 \pm 1,34$ \\
\hline & Total & 109 & $23,7 \pm 13,2$ & $118,9 \pm 66,5$ & $4,26 \pm 1,52$ \\
\hline
\end{tabular}

$H D L=$ do inglês: lipoproteína de alta densidade; $L D L=$ do inglês: lipoproteína de baixa densidade; $V L D L=$ do inglês: lipoproteína de muito baixa densidade; $I A=$ índice aterogênico.

O coeficiente de determinação $\left(\mathrm{R}_{\text {ajustado }}^{2}\right.$ Tabela 3$)$ indica que o NAF e as atividades para o controle do estresse têm baixa $(\mathrm{p}>0,05)$ explicação na variação do perfil lipídico. Por exemplo, se o sujeito sai da categoria "NAF ruim" para a "NAF bom", o colesterol total diminui somente $1,7 \%$, o HDL $1 \%$ e assim sucessivamente. 
Tabela 3. Análise de variância a dois fatores do perfil lipídico em relação ao nível de atividade física (NAF) e controle do estresse (ctrl stress).

\begin{tabular}{|c|c|c|c|c|c|c|}
\hline \multirow{2}{*}{ Fator } & \multicolumn{3}{|c|}{ Colesterol total } & \multicolumn{3}{|c|}{ HDL } \\
\hline & $\mathrm{F}$ & $p$ & Poder do teste & $\mathrm{F}$ & $p$ & Poder do teste \\
\hline NAF & 1,496 & 0,225 & 0,226 & 0,951 & 0,333 & 0,161 \\
\hline Ctrl stress & 0,266 & 0,607 & 0,080 & 0,261 & 0,611 & 0,080 \\
\hline \multirow[t]{2}{*}{ NAF * Stress } & 1,283 & 0,261 & 0,201 & 0,090 & 0,765 & 0,060 \\
\hline & \multicolumn{3}{|c|}{ LDL } & \multicolumn{3}{|c|}{ VLDL } \\
\hline NAF & 1,582 & 0,213 & 0,237 & 0,822 & 0,368 & 0,145 \\
\hline Ctrl stress & 0,011 & 0,916 & 0,051 & 0,514 & 0,476 & 0,109 \\
\hline \multirow[t]{2}{*}{ NAF * Stress } & 1,263 & 0,265 & 0,198 & 0,044 & 0,835 & 0,055 \\
\hline & \multicolumn{3}{|c|}{ Triglicérides } & \multicolumn{3}{|c|}{ Índice aterogênico } \\
\hline NAF & 0,874 & 0,353 & 0,152 & 1,545 & 0,218 & 0,232 \\
\hline Ctrl stress & 0,321 & 0,573 & 0,086 & 0,095 & 0,759 & 0,061 \\
\hline NAF * Stress & 0,004 & 0,947 & 0,050 & 0,002 & 0,965 & 0,050 \\
\hline
\end{tabular}

$\mathrm{HDL}=$ do inglês: lipoproteína de alta densidade; $F=$ teste $F ; p=$ probabilidade; $\mathrm{LDL}=$ do inglês: lipoproteína de baixa densidade; $V L D L=$ do inglês: lipoproteína de muito baixa densidade; $R^{2}$ : colesterol total $=-0,017$; $\mathrm{HDL}=-0,010 ; \mathrm{LDL}=-0,010 ; \mathrm{VLD}=-0,009$; triglicérides $=-0,017$; Índice aterogênictcoldo $=-0,008$.

\section{DISCUSSÃO}

No presente estudo buscou-se observar as possíveis diferenças no perfil lipídico de homens, de acordo com o NAF e de controle do estresse, baseado em indicativos prévios de que existe uma possível relação entre a atividade física e diversos fatores de risco à saúde, tendo os indivíduos sedentários, perfil lipídico inadequado $o^{3-5}$, além de uma possível influência positiva da prática de atividades físicas sobre as condições de estresse ${ }^{8}$ e deste sobre o perfil lipídico ${ }^{19}$. Neste contexto, foi analisado se o NAF e o controle de estresse, estimados por questionários, são capazes de diferenciar sujeitos quanto ao perfil lipídico.

As análises revelaram que as variáveis do perfil lipídico não diferiram ( $p>0,05)$ entre os homens com NAF bom e ruim, e que possuem ou não controle do estresse. A chance do NAF e do ctrl stress influenciarem o perfil lipídico foi baixa ( $\mathrm{p}>0,05$ ). Para o NAF oscilou de 14,5\% (VLDL) a $23,7 \%$ (LDL) e, para o ctrl stress de 5,1\% (LDL) a 10,9\% (VLDL). Tanto o NAF quanto o ctrl stress tiveram explicação muito baixa $(p>0,05)$ na variação do perfil lipídico, sendo a mais alta de 1,7\%. Em média, aqueles com NAF bom e com ctrl stress têm a chance de apresentar seu colesterol total e triglicérides somente $1,7 \%$ mais baixo do que aqueles com NAF ruim e sem ctrl stress. Em consequência, não houve $(\mathrm{p}>0,05)$ interação entre o NAF e o controle do estresse, revelando que o seu efeito global pode ser generalizado para todos os perfis lipídicos. Ainda, diferente de evidência existente $^{8}$, o NAF não exerceu nenhuma influência sobre o ctrl stress.

Este fato não era esperado, pois estudos prévios indicaram que indivíduos ativos apresentam um perfil lipídico favorável frente aos sedentários ${ }^{3-5,20,21}$, além das condições de estresse interferirem nas variáveis lipídicas ${ }^{19,22}$. Nesse contexto, parece que os sujeitos que possuem NAF bom 
e que realizam atividades de ctrl stress estão expostos da mesma forma aos fatores de risco cardiovascular (aqui analisados) que seus pares com comportamentos opostos.

No entanto, se hipotetiza que a evidência no presente estudo não seja uma falta de relação entre causa e efeito, mas uma possível decorrência das características dos instrumentos usados para classificar os sujeitos quanto ao NAF e ao ctrl stress. Em um grupo de homens estratificados da mesma forma que neste, e usado o mesmo questionário para estimar o NAF, fora observado uma concordância de $58,8 \%$ entre o questionário e o consumo máximo de oxigênio $\left(\mathrm{VO}_{2} \mathrm{máx}\right)^{11}$. Destaca-se que, $94,1 \%$ da amostra apresentou $\mathrm{VO}_{2}$ máx bom, ou adequado à saúde $\left(\geq 44 \mathrm{ml} / \mathrm{Kg} / \mathrm{min}^{-1}\right)$ e, $35,3 \%$ dela fora classificada com NAF ruim ${ }^{11}$. Ainda neste mesmo estudo, noutro grupo de homens, foi observado que somente $46,5 \%$ deles apresentaram $\mathrm{VO}_{2}$ máx bom, enquanto que, pelo questionário, $81,4 \%$ apresentaram NAF bom. Então, sujeitos com melhores $\mathrm{VO}_{2}$ máx tendem a subestimar seu NAF quando analisados via QAFH. Isto demonstra que este questionário apresenta variabilidade muito grande em classificar acuradamente grupos distintos. Ainda, estudos indicam que há relação inversa entre o perfil lipídico e o $\mathrm{VO}_{2}$ máx e que este é mais fortemente associado aos fatores de risco cardiovascular do que o $\mathrm{NAF}^{13,23}$. Desta forma, parece que o questionário usado no presente estudo não é suficientemente adequado para indicar o NAF e, a partir daí, fazer inferências relacionadas aos principais fatores de risco cardiovascular (ex.: perfil lipídico).

Diferentemente dos estudos que analisaram as relações entre o perfil lipídico e algum tipo de estresse, no presente foram consideradas as atividades realizadas, ou não, para o ctrl stress. Desta forma, o fato de o sujeito realizar, ou não, as atividades para o ctrl stress, necessariamente não significa que ele possui estresse.

A discussão entre o perfil lipídico e o ctrl stress é limitada devido ao fato de não haver estudos que utilizassem o questionário do "Perfil do Estilo de Vida Individual" com fins de analisar atributos/comportamentos caracterizados via este, em relação ao perfil lipídico e, nem mesmo com outro instrumento de referência (padrão ouro). No entanto, se observa com frequência o uso do mesmo e do QAFH com a finalidade de caracterizar os comportamentos em relação à saúde ${ }^{24,25}$. Diante disto, e por ser observada relação entre o NAF, o estresse e o perfil lipídico ${ }^{3,4,19,22,26}$, parece que estes instrumentos possuem fragilidade, principalmente, quando do seu uso para fazer inferências relacionadas ao principais fatores de risco cardiovascular (ex.: perfil lipídico).

Alguns aspectos podem limitar as generalizações deste estudo, dentre eles se destacam: 1) O uso de um kit comercial, não comumente adotado como "padrão ouro" no método de espectrofotometria enzimática e, o espectrofotômetro usado ser semiautomático, o que pode aumentar as chances de erro. $\mathrm{O}$ fato de ter sido usado este kit pode diminuir a validade dos resultados. No entanto, ele é o mais usado no Centro-Oeste do Brasil, o que justifica o seu uso neste estudo, uma vez que a amostra reside nesta 
região. Além do que a sua sensibilidade é de $1 \mathrm{mg} / \mathrm{dL}$, com coeficiente de variação para a reprodutibilidade, oscilando de $1,26 \%$ a $2,76 \%$, e de 0,79 $\%$ a 1,04 \% para a repetitividade. 2) O não controle dos hábitos alimentares, haja vista este afetar o perfil lipídico. Todavia, cabe salientar que a amostra fazia três refeições diárias (café da manhã, almoço e lanche) no local do trabalho, o que pode tornar a alimentação deles, de certa forma, similar. 3) Os questionários para o NAF e ctrl stress (previamente discutido).

Mesmo diante destas limitações o estudo não perde sua validade, pois outros $^{4,5,17,20,24}$ apontam que o NAF e o estresse têm relação com o perfil lipídico e neste estudo tal fato não ocorreu, possivelmente em decorrência dos questionários usados. Assim, este estudo reforça a necessidade de se buscar o desenvolvimento e validação de instrumentos realmente acurados para quantificar estes atributos, e realça a necessidade de evitar o seu uso em triagens com fins de adoção de políticas públicas direcionadas à promoção da saúde, principalmente, no que tange àquelas direcionadas aos fatores de risco cardiovascular. Ainda, os resultados obtidos também subsidiam a elaboração de hipóteses para os possíveis estudos com fins de analisar as variáveis em questão, porém também considerando o $\mathrm{VO}_{2}$ máx e a presença diagnosticada de estresse.

Tanto o NAF quanto o estresse podem causar algum impacto nas variáveis lipídicas. Reforça-se o envolvimento frequente com práticas de atividades físicas, principalmente, de intensidade moderada ou vigorosa, pelo seu importante papel em muitas condições de saúde, com impacto positivo, para a redução da prevalência da maioria das doenças crônicas não transmissíveis ${ }^{27}$.

\section{CONCLUSÕES}

Em termos gerais, diretamente os resultados do presente estudo possibilitam inferir que o perfil lipídico de homens não difere entre aqueles com nível de atividade física bom e ruim e, que fazem, ou não, atividades de controle do estresse. Tanto o NAF quanto o controle de estresse tiveram uma chance muito pequena em influenciar as variáveis do perfil lipídico. O efeito global do NAF e do controle de estresse pode ser generalizado para todos os perfis lipídicos, quando usados os mesmos instrumentos do presente estudo. Estes achados têm implicações importantes quando o propósito for averiguar se os sujeitos alcançaram, ou não, o NAF bom. Hipotetiza-se que tais achados são devidos a possível falta de acuracidade na obtenção do NAF e do controle de estresse. Recomenda-se muita cautela ao empregar os mesmos instrumentos do presente estudo, principalmente, ao tomar os resultados decorrentes como referência para adoção de políticas públicas para promoção e manutenção da saúde.

\section{REFERÊNCIAS BIBLIOGRÁFICAS}

1. Löllgen H, Böckenhoff A. Knapp G. Physical activity and all-cause mortality: an updated meta-analysis with different intensity. Int J Sports Med 2009;30(3):213-24.

2. Bandeira de Sá NN, Moura EC. Fatores associados à carga de doenças da síndrome metabólica entre adultos brasileiros Cad Saúde Pública 2010;26(9):1853-62. 
3. Morris JN, Heady JA, Raffle PAB, Roberts CG, Parks JW. Coronary heart disease and physical activity of work. Lancet 1953;2(6796):1111-20.

4. Blair SN, Kampert JB, Kohl HW, Barlow CE, Macera CA, Paffenbarger RS, et al. Influences of cardiorespiratory fitness and other precursors on cardiovascular disease and all-cause mortality in men and women. JAMA 1996;276(3):205-10.

5. Jurca R, Michael JL, Timothy SC, Conrade PE, Shannon JF, Carolyn EB, et al. Associations of muscle strength and aerobic fitness with Metabolic Syndrome in men. Med Sci Sports Exerc 2004;36(8):1301-7.

6. Barreto AC, Barbosa-Branco A. Influência da atividade física sistematizada no estresse e na fadiga dos trabalhadores do restaurante universitário de da Universidade de Brasília. Rev Bras Ativ Fis Saúde 2000;5(2):23-9.

7. Peluso MAM, Andrade LHSG. Physical activity and mental health: the association between exercise and mood. Clinics 2005;1(60):61-70.

8. Nunomura M, Teixeira LAC, Caruso MRF. Nível de estresse em adultos após 12 meses de prática regular de atividade física. REMEFE 2004;3(3):125-34.

9. Siqueira FV, Facchini LA, Piccini RX, Tomasi E, Thumé E, Silveira DS, et al. Atividade física em adultos e idosos residentes em áreas de abrangência de unidades básicas de saúde de municípios das regiões Sul e Nordeste do Brasil. Cad Saúde Pública 2008;24(1):39-54.

10. Knuth AG, Malta DC, Dumith SC, Pereira CA, Morais Neto OL, Temporão JG, et al. Prática de atividade física e sedentarismo em brasileiros: resultados da Pesquisa Nacional por Amostra de Domicílios (PNAD) 2008. Ciênc saúde coletiva 2011;16(9):3697-705.

11. Glaner MF. Concordância de questionários de atividade física com a aptidão cardiorrespiratória. Rev Bras Cineantropom Desempenho Hum 2007;9(1):61-6.

12. Ainsworth BE, Bassett DR, Jr., Strath SJ, Swartz AM, O’Brien WL, Thompson RW, et al. Comparison of three methods for measuring time spent in physical activity. Med Sci Sports Exerc 2000;32(9)S457-S64.

13. Hurtig-Wennlöf A, Ruiz JR, Harro M, Sjöström M. Cardiorespiratory fitness relates more strongly than physical activity to cardiovascular disease risk factors in healthy children and adolescents: the European Youth Heart Study. Eur J Cardiovasc Prev Rehabil 2007;14(4):575-81.

14. Glaner MF, Pelegrini A, Nascimento TBR. Perímetro do abdômen é o melhor indicador antropométrico de riscos para doenças cardiovasculares. Rev Bras Cineantropom Desempenho Hum 2011;13(1):1-7.

15. Hu G, Tuomilehto J, Silventoinen K, Sarti C, Mannisto S, Jousilahti P. Body mass index, waist circumference, and waist-hip ratio on the risk of total and type-specific stroke. Arch Intern Med 2007;167(13)1420-7.

16. Lohman TG, Roche AF, Martorell R. Anthropometric standartization reference manual. Champaign: Human Kinetics Books, 1991.

17. Nahas MV. Atividade Física, saúde e qualidade de vida: conceitos e sugestões para um estilo de vida. Londrina: Midiograf, 2003.

18. Friedewald WT, Levy RI, Fredrickson DS. Estimation of the concentration of low-density lipoprotein cholesterol in plasma without use of preparative ultracentrifuge. Clin Chem 1972;18(6):499-502.

19. Ronsein GE, Dutra RL, Silva EL, Martinello F, Hermes EM, Balen G, et al. Influência do estresse nos níveis sanguíneos de lipídios, ácido ascórbico, zinco e outros parâmetros bioquímicos. Acta Bioquím Clín Latinoam 2004;38(1):39-46.

20. Verdaet D, Dendale B, Bacquer D, Delanghe J, Block P, De Backer G. Association between leisure time physical activity and markers of chronic inflammation related to coronary heart disease. Atherosclerosis 2004;176(2):303-10.

21. Chitra U, Reddy NK, Balakrishna N. Role of lifestyle variables on the lipid profile of selected South Indian subjects. Indian Heart J 2012;64(1):28-34.

22. Rozanski A, Blumenthal JA, Kaplan J. Impact of psychological factors on the pathogenesis of cardiovascular disease and implications for therapy. Circulation 1999;99(16): 2192-2217. 
23. Petrović-Oggiano G, Damjanov V, Gurinović M, Glibetić M. Physical activity in prevention and reduction of cardiovascular risk. Med Pregl 2010;63(3-4):200-7.

24. Viana MS, Andrade A, Back AR, Vasconcellos DIC. Nível de atividade física, estresse e saúde em bancários. Motricidade 2010:6(1):19-32.

25. Silva RS, Silva I, Silva RA, Souza L, Tomasi E. Atividade física e qualidade de vida. Ciênc saúde coletiva 2010:15(1):115-20.

26. Costa MB, Guércio NMS, Costa HFC. Possível relação entre estresse ocupacional e síndrome metabólica. HU Revista 2011;37(1):87-93.

27. World Health Organization/WHO. Global Recommendations on Physical Activity for Health. 2011. Available from: <http://www.who.int/dietphysicalactivity/ factsheet_recommendations/en/index.html $>$ [2012 jul 12].
Endereço para correspondência

Maria Fátima Glaner

UEL / CEFE / DEF

Rodovia Celso Garcia Cid - PR 445,

$\mathrm{km} 380$

Campus Universitário.

Cx. Postal 10.011

CEP 86057-970 - Londrina - PR / Brasil E-mail: mfglaner@gmail.com 